\title{
Inhibition of autophagy enhances the targeted therapeutic effect of sorafenib in thyroid cancer
}

\author{
HEQING YI ${ }^{1}$, TING YE ${ }^{1}$, MINGHUA GE ${ }^{2}$, MENGDI YANG ${ }^{1}$, LIJUN ZHANG ${ }^{1}$, \\ SHUI JIN ${ }^{1}$, XUEMEI YE ${ }^{1}$, BIN LONG ${ }^{1}$ and LINFA LI ${ }^{1}$ \\ Departments of ${ }^{1}$ Nuclear Medicine and ${ }^{2} \mathrm{Head}$ and Neck Surgery, \\ Zhejiang Cancer Hospital, Hangzhou, Zhejiang 310021, P.R. China
}

Received July 9, 2017; Accepted November 23, 2017

DOI: 10.3892/or.2017.6118

\begin{abstract}
The multi-target kinase inhibitor sorafenib has been approved for the treatment of patients with advanced differentiated thyroid cancer. However, different sensitivities to sorafenib have been observed, and few patients have benefited from sorafenib treatment in the long term. In the event of acquired resistance to sorafenib it is not beneficial to continue treatment in most patients. Autophagy can be induced in a variety of cancer treatments and plays an important role in cancer treatment. The role of autophagy in sorafenib treatment of thyroid cancer has not been fully demonstrated. The present study investigated whether autophagy is activated by sorafenib during the treatment of thyroid cancer, examined the underlying mechanisms, and explored potential strategies to enhance the therapeutic sensitivity of sorafenib. Chloroquine (CQ) is an autophagy inhibitor that has been reported to increase sensitivity to various cancer treatments. Thyroid cancer xenograft model mice were treated with sorafenib, $\mathrm{CQ}$, or a combination of sorafenib and CQ. We observed that CQ or sorafenib treatment suppressed tumor growth, while mice treated with the combination of sorafenib and CQ displayed significantly reduced tumor growth compared with those treated with sorafenib or CQ alone. Western blotting results indicated that sorafenib concurrently inhibited the activities of the MAPK and AKT/mTOR pathways in thyroid cancer. Autophagy was activated by sorafenib in thyroid cancer, both in vitro and in vivo, which was at least in part due to suppression of the AKT/mTOR pathway. Combination treatment including CQ could inhibit the autophagic flux induced by sorafenib. Silencing the key autophagy gene ATG5 using small interfering RNA also increased the anticancer effect of sorafenib. In summary, the present study revealed that inhibition of autophagy enhances the anticancer effect of sorafenib, and
\end{abstract}

Correspondence to: Dr Linfa Li, Department of Nuclear Medicine, Zhejiang Cancer Hospital, 1st Banshan East Road, Hangzhou, Zhejiang 310021, P.R. China

E-mail: pet-ct001@163.com

Key words: thyroid cancer, sorafenib, autophagy, iodine resistance the combination of CQ with sorafenib treatment represents a potential therapeutic strategy for treating advanced differentiated thyroid cancer.

\section{Introduction}

A marked increase in the number of cases of thyroid cancer has been consistently observed in several countries, including China. It is estimated that approximately 90,000 new thyroid cancer cases were diagnosed in China in 2015 (1). More than $90 \%$ of thyroid cancer cases are differentiated thyroid cancers (DTC). Some DTC patients with distant metastasis are resistant to ${ }^{131}$ iodine. Traditional cancer treatments are usually not effective for ${ }^{131}$ iodine-resistant DTC patients, who have shorter overall survival times than ${ }^{131}$ iodine-responsive DTC patients $(2,3)$.

The biological behaviors of tumors are driven by oncogenes (4). The MAPK and PI3K/AKT pathways are two important pathways driving thyroid tumor development and progression (5). The activities of BRAF and RAS, two key components of the MAPK pathway, affect cancer cell proliferation and resistance to anticancer treatment (6). Multiple genes in the MAPK pathway are targeted by sorafenib, a kinase inhibitor $(7,8)$. Sorafenib was recently approved as the first targeted drug for advanced metastatic DTC by the Chinese Food and Drug Administration. However, sorafenib only improves progression-free survival for $\sim 5$ months compared with patients on a placebo (9), and the majority of patients acquire resistance to sorafenib after 1 or 2 years of administration, which is an obstacle in clinical practice (10).

Autophagy is an evolutionarily conserved process in which cytoplasmic materials are delivered to lysosomes for degradation (11). The relationship between autophagy and cancer is complex due to the dual roles of autophagy in tumor progression and cancer therapy $(11,12)$. Several studies have reported that sorafenib induced autophagy in cancer cells (13), and it has been reported that autophagy can be induced by sorafenib in a dose- and time-dependent in several hepatocellular carcinoma (HCC) cell lines $(14,15)$. It has also been observed that sorafenib modulated the expression of LC3, Beclin-1, Atg5 and Atg12, and decreased the expression of p62 in HCC cells in vitro $(16,17)$. Several studies have reported that sorafenib inhibited AKT phosphorylation in renal cancer cells and 
HCC, as reviewed by Prieto-Dominguez et al (13). In addition, inhibition of AKT enhanced sorafenib-induced autophagy, and switched protective autophagy to autophagic cell death in HCC (17). Although the role of autophagy in cancer has been extensively investigated, its role in the treatment of thyroid cancer by sorafenib has not been clearly determined.

We previously observed that sorafenib exerted a therapeutic effect by targeting the MAPK and AKT/mTOR pathways. However, the AKT/mTOR pathway is a negative regulator of autophagy and the role of autophagy in cancer treatment is complex $(17,18)$. In this study, we aimed to investigate whether autophagy was induced by sorafenib treatment in thyroid cancer cells and to elucidate whether autophagy inhibition increases or reduces sorafenib-induced thyroid cancer cell death.

\section{Materials and methods}

Cell culture and materials. The human thyroid cancer cell lines $8505 \mathrm{C}$ and FTC133 were cultured at $37^{\circ} \mathrm{C}$ in Dulbecco's modified Eagle's medium (Gibco; Thermo Fisher, Shanghai, China) supplemented with $10 \%$ fetal bovine serum (Gibco; Thermo Fisher), $100 \mathrm{U} / \mathrm{ml}$ penicillin and $100 \mathrm{~g} / \mathrm{ml}$ streptomycin in a humidified incubator with $5 \% \mathrm{CO}_{2}$. Antibodies against Beclin-1 (mouse, 1:1,000, SC-10086), GAPDH (mouse, 1:1,000, SC-51907), p-ERK (mouse, 1:200, SC-7383), ERK (rabbit, 1:1,000, SC153) and AKT (mouse, 1:1,000, SC-5298) were purchased from Santa Cruz Biotechnology, Inc. (Dallas, TX, USA), and the anti-p-AKT (Ser473) antibody was purchased from Abcam (rabbit, 1:200, Cambridge, Massachusetts, USA). Antibodies against cleaved-caspase-3 (rabbit, 1:1,000, \#9661), p62 (rabbit, 1:1,000, \#88588), LC3 (rabbit, 1:1,000, \#12741), p70s6 (rabbit, 1:1,000, \#2708) and p-p70s6 (rabbit, 1:500, \#2211) were purchased from Cell Signaling Technology, Inc. (Danvers, MA, USA). BEZ-234 was purchased from Selleck (Shanghai, China), and sorafenib was generously donated by the Bayer Company (Shanghai, China). Cells were transfected with scrambled (negative control) small interfering (si)RNA (5'-UUCUCCGAACG UGUCACGUTT-3' and 5'-ACGUGACACGUUCGGAGA ATT-3'),AKT siRNA(5'-GACGGGCACAUUAAGAUCATT-3' and 5'-UGAUCUUAAUGUGCCCGUCTT-3'), or ATG5 siRNA (5'-GUCCAUCUAAGGAUGCAAUTT-3' and 5'-AUUGCAU CCUUAGAUGGACTT-3') and purchased from GenePharma (Shanghai, China). Transfection was performed using Lipofectamine $^{\mathrm{TM}} 2000$ (Invitrogen, Shanghai, China).

Mouse xenograft. Nude BALB/c mice (5-6 weeks old; male; Animal Core Facility of Nanjing Medical University, Nanjing, China) were used to generate xenograft tumor models. All animal studies complied with the management rules of the National Health and Family Planning Commission of China and were approved by the Ethics Committee of Zhejiang Cancer Hospital. A suspension of $5 \times 10^{6} 8505 \mathrm{C}$ or FTC133 cells in $100 \mu \mathrm{l}$ PBS was inoculated subcutaneously into the right flanks of the mice. When the tumor sizes averaged approximately $5 \times 5 \mathrm{~mm}$, the mice were randomly divided into four groups that were matched for tumor volume, and treatment was initiated (6 mice per treatment group). Treatment groups were as follows: the vehicle control group (DMSO, $\leq 0.1 \%$ ), the chloroquine group (CQ; Sigma-Aldrich, Shanghai, China), the sorafenib group, and the sorafenib + CQ group. Sorafenib was administered at doses of $30 \mathrm{mg} / \mathrm{kg}$ every day for 21 days. CQ was administered at doses of $60 \mathrm{mg} / \mathrm{kg}$ every day for 21 days. In the sorafenib + CQ group, sorafenib was administered 20 min after CQ administration. All drugs were administered via intraperitoneal injection. A caliper was used to assess the tumors every 4 days. After 21 days of treatment, the mice were sacrificed and the tumor tissues were harvested for study. The mice were sacrificed by anesthetizing with an intraperitoneal injection of $0.8 \%$ pentobarbital sodium $(60 \mathrm{mg} / \mathrm{kg})$, followed by cervical dislocation. All efforts were made to reduce pain experienced by the mice.

Western blot analysis. Tumor tissues or cell pellets were lysed in RIPA lysis buffer and a protease inhibitor cocktail (Sigma-Aldrich). Total protein samples were separated by $10 \%$ or $12 \%$ sodium dodecyl sulfate polyacrylamide gel electrophoresis (SDS-PAGE) and transferred onto nitrocellulose membranes. The membranes were blocked with 5\% non-fat dry milk and incubated overnight with the various primary antibodies at $4^{\circ} \mathrm{C}$. Goat anti-mouse (sc-2005) or anti-rabbit (sc-2004) antibodies were purchased from Santa Cruz Biotechnology, Inc. and diluted to $1: 1,000$ in 5\% skim milk, Tris- $\mathrm{HCl}(\mathrm{pH} 7.5)$ and $0.1 \%$ Tween-20. The immunoblots were subsequently washed and incubated with the goat anti-mouse or anti-rabbit antibody for $1 \mathrm{~h}$ at room temperature. The results were visualized using chemiluminescence reagent (sc-2048, Santa Cruz Biotechnology, Inc.).

$R N A$ extraction and reverse transcription-quantitative $P C R$ $(R T-q P C R)$. Total RNA was isolated using an RNA Prep Pure kit (Tiangen, Beijing, China), according to the manufacturer's instructions. The synthesis of cDNA was performed with HiScript II Q RT Super Mix for qPCR (+gDNA wiper) (Vazyme Biotech Co., Nanjing, China). qPCR was performed with an AceQ qPCR SYBR Green Master Mix (without ROX) (Vazyme Biotech Co.). GAPDH was used as an internal control. Primer sequences used in the qPCR analysis were purchased from GenePharma (Shanghai, China), and the sequences were as follows: AKT, 5'-CCAACACCTTCATCA TCC-3' and 5'-CTCCTCCTCCTGCTTCTT-3'; ATG5, 5'-GCAGATGGACAGTTGCACACA-3' and 5'-TTTCCCCAT CTTCAGGATCAA-3'; and GAPDH, 5'-CGGAGTCAACGGA TTTGGTCGTAT-3' and 5'-AGCCTTCTCCATGGTGGTGA AGAC-3'. Melt curve was made to determine the optimal condition $\left(95^{\circ} \mathrm{C} 15 \mathrm{sec}, 60^{\circ} \mathrm{C} 30 \mathrm{sec}, 95^{\circ} \mathrm{C} 15 \mathrm{sec}\right)$. The PCR protocol is as follows: denaturation $95{ }^{\circ} \mathrm{C}$ for $5 \mathrm{~min}$, then 40 amplification cycles $\left(95^{\circ} \mathrm{C}\right.$ for $5 \mathrm{sec}$ and $60^{\circ} \mathrm{C}$ for $31 \mathrm{sec}$, at a ramp-rate of $\left.1.6^{\circ} \mathrm{C} / \mathrm{sec}\right)$.

Apoptosis assay. 8505C and FTC133 cells (2x105/well) were seeded into a 6-well plate. After treatment with ATG5Ri mimic, sorafenib $(10 \mu \mathrm{M}), \mathrm{BEZ}-235(0.8 \mu \mathrm{M})$ or combined sorafenib + BEZ-235, the cells were harvested and analyzed for apoptosis by Annexin $\mathrm{V}$ and propidium iodide staining, using an FITC Annexin V Apoptosis Detection kit (Life Technologies, Waltham, MA USA), according to the manufacturer's instructions, and a flow cytometer (FACS, Beckman Coulter, Miami, FL, USA). 
$\mathbf{A}$

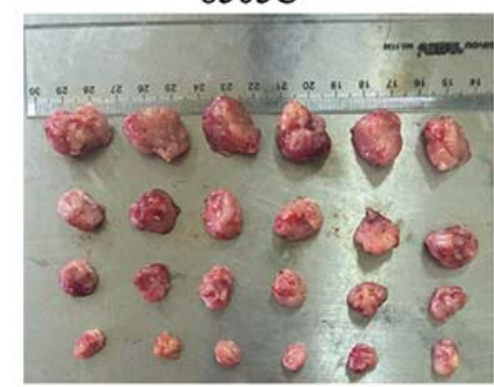

C

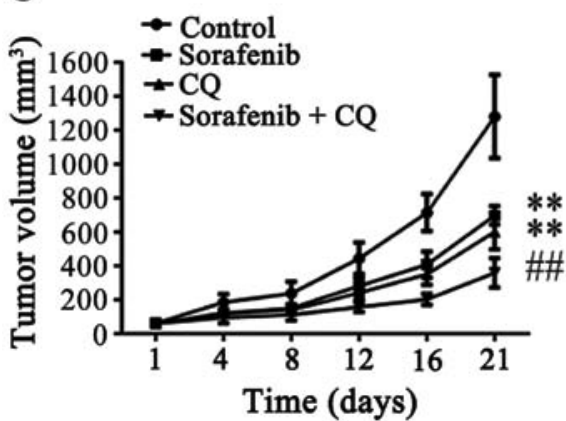

B

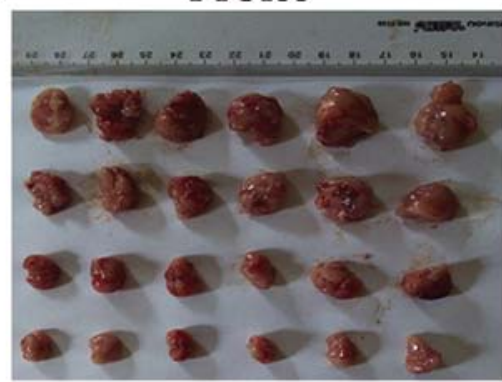

D

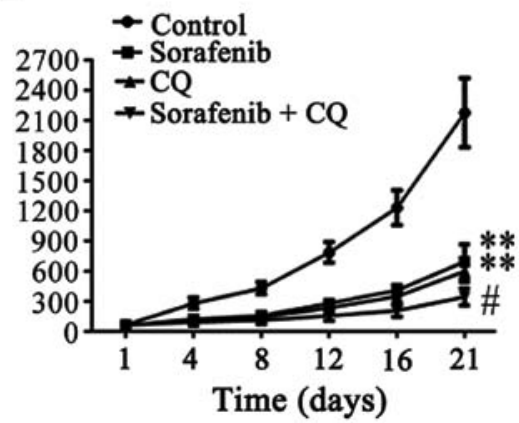

Figure 1. Effects of combined CQ and sorafenib treatment in vivo. (A and B) Subcutaneous tumors (8505C or FTC133 cells) were established in mice as described in Materials and methods. After 21 days of treatment, the mice were sacrificed and tumor tissues were harvested for study. From top to bottom the groups were: control, sorafenib, CQ, sorafenib + CQ. (C and D) Tumor volumes were assessed every 4 days and calculated using the following formula: volume $=\left(\right.$ length $\mathrm{x}$ width $\left.{ }^{2}\right) / 2 .{ }^{* *} \mathrm{P}<0.01$ vs. the control group; ${ }^{*} \mathrm{P}<0.05$ and ${ }^{\# \#} \mathrm{P}<0.01$ vs. sorafenib or $\mathrm{CQ}$ alone.

Immunohistochemistry (IHC). Paraffin-embedded mouse tumor samples were sliced into 5- $\mu \mathrm{m}$ sections and mounted on microscope slides. Slides were incubated at $37^{\circ} \mathrm{C}$ overnight for deparaffinization. The slides were dipped in dimethylbenzene three times (10 $\mathrm{min} / \mathrm{dip})$. The slides were then immediately dipped in 100, 95, 90, 80, 70 and 50\% alcohol continuously for $2 \mathrm{~min}$ at each percentage (\%) of alcohol. Antigen retrieval was performed by heating for $15 \mathrm{~min}$ in $0.01 \mathrm{M}$ sodium citrate buffer ( $\mathrm{pH}$ 6.0). The slides were washed in TBS three times (5 min/wash), treated with $3 \% \mathrm{H}_{2} \mathrm{O}_{2}$ and blocked with $5 \%$ BSA for $20 \mathrm{~min}$, then incubated with $50 \mu \mathrm{l}$ cleaved-caspase-3 (1:200 dilution) at $4{ }^{\circ} \mathrm{C}$ overnight. Subsequently, the slides were washed, and incubated with a mouse anti-rabbit antibody for $1 \mathrm{~h}$ at room temperature. A DAB horseradish peroxidase color development kit (ZSGB-BIO, Beijing, China) was used to detect positive staining. The slides were also counterstained with hematoxylin for $25 \mathrm{sec}$, washed under running water for $3 \mathrm{~min}$, then washed with distilled water for $1 \mathrm{~min}$ followed by dipping in 50, 70, 80, 90 and $100 \%$ alcohol continuously for 1 min each. Finally, the slides were dipped in dimethylbenzene for $3 \mathrm{~min}$, air-dried and mounted with Permount ${ }^{\mathrm{TM}}$ Mounting Medium (eBioscience, San Diego, CA, USA).

Cell proliferation and viability assays. 8505C and FTC133 cells were plated at a density of $1 \times 10^{4}$ cells/well in 96 -well microtiter plates, and each plate was incubated for $24 \mathrm{~h}$ at $37^{\circ} \mathrm{C}$ in $5 \% \mathrm{CO}_{2}$. Following treatment, the absorbance of the contents of each well was assessed at $470 \mathrm{~nm}$ following the use of a CCK-8 kit (Beyotime, Guangzhou, China).

Statistical analysis. Data are presented as the mean \pm standard error of the mean. The results were analyzed using
One-way ANOVA, and multiple comparison using StudentNewman-Keuls (SNK) and least significant difference (LSD) tests. $\mathrm{P}<0.05$ was considered to indicate a statistically significant difference.

\section{Results}

CQ increases the therapeutic effect of sorafenib and suppresses tumor growth in xenograft models. To investigate whether autophagy influences the therapeutic efficacy of sorafenib in vivo, 5-6-week-old male BALB/c nude mice were used as an animal model. CQ is a potential autophagy inhibitor that has been used to regulate autophagy in vivo and in vitro $(19,20)$. Mice were randomly divided into control, sorafenib (30 mg/kg/day), CQ (60 mg/kg/day), or sorafenib + CQ groups (n=6/group) at day 7 after subcutaneous injection of $5 \times 10^{6}$ cancer cells. Tumor volumes were monitored every 4 days, and the mice were sacrificed after 21 days of treatment for isolation of tumor tissues (Fig. 1A and B). Compared with the tumor sizes in the control group $\left(1,280.93 \pm 245.27 \mathrm{~mm}^{3}\right.$ in $8505 \mathrm{C}$ xenograft mice; $2,176.57 \pm 343.82 \mathrm{~mm}^{3}$ in FTC133 xenograft mice), the tumor volumes significantly decreased after administration of CQ or sorafenib alone $(602.36 \pm 101.18$ and $698.61 \pm 54.53 \mathrm{~mm}^{3}$ in $8505 \mathrm{C}$ xenograft mice, respectively; $600.42 \pm 107.33$ and $697.07 \pm 171.34 \mathrm{~mm}^{3}$ in FTC133 xenograft mice, respectively) (Fig. $1 \mathrm{C}$ and D), while the combined treatment of CQ + sorafenib significantly suppressed tumor volumes compared with CQ or sorafenib treatments alone in the $8505 \mathrm{C}$ and FTC133 xenograft mice $(358.99 \pm 86.54$ and $344.36 \pm 84.36 \mathrm{~mm}^{3}$, respectively) (Fig. 1C and D). There were no significant differences between $\mathrm{CQ}$ and sorafenib treatment groups in $8505 \mathrm{C}$ or FTC133 xenograft mice. 
$\mathbf{A}$

LC3

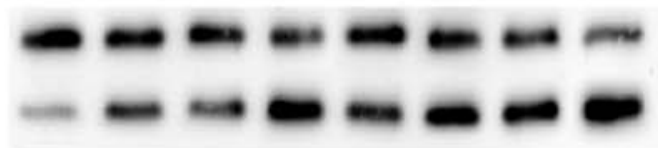

p62

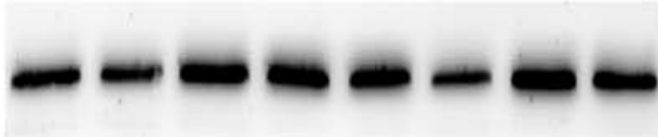

GAPDH

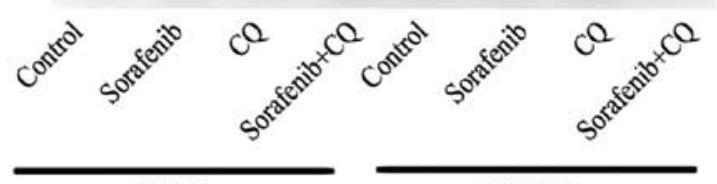

$8505 \mathrm{C}$

D

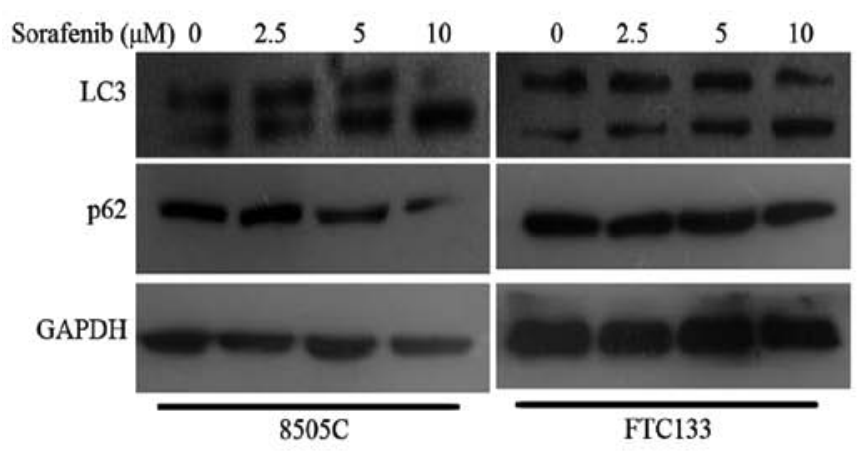

G
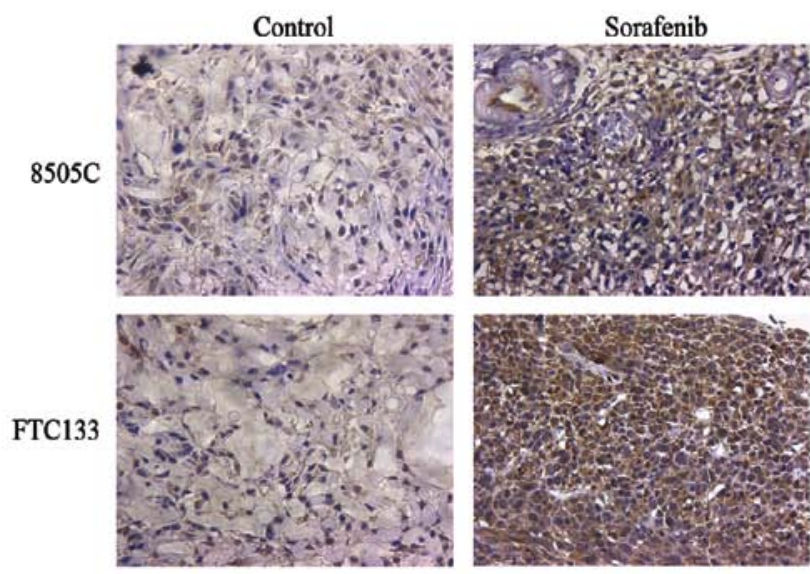

FTC133

B

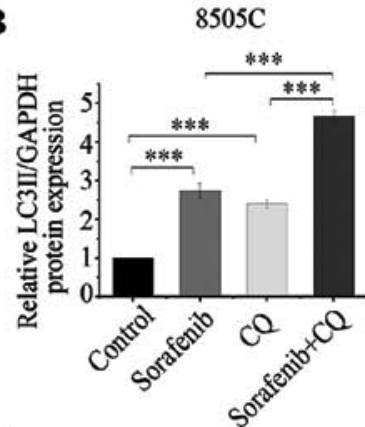

C

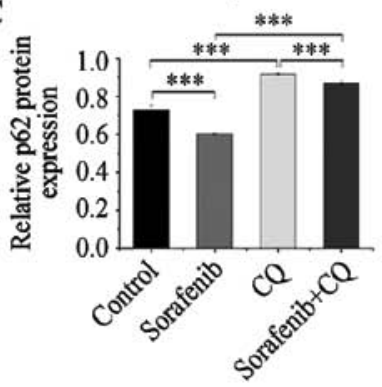

$\mathbf{E}$

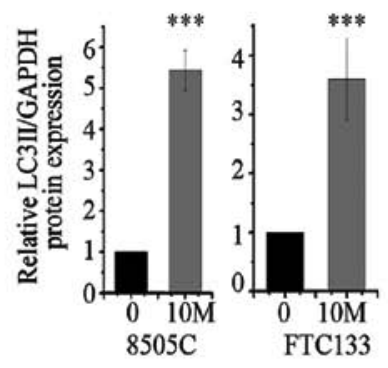

CQ
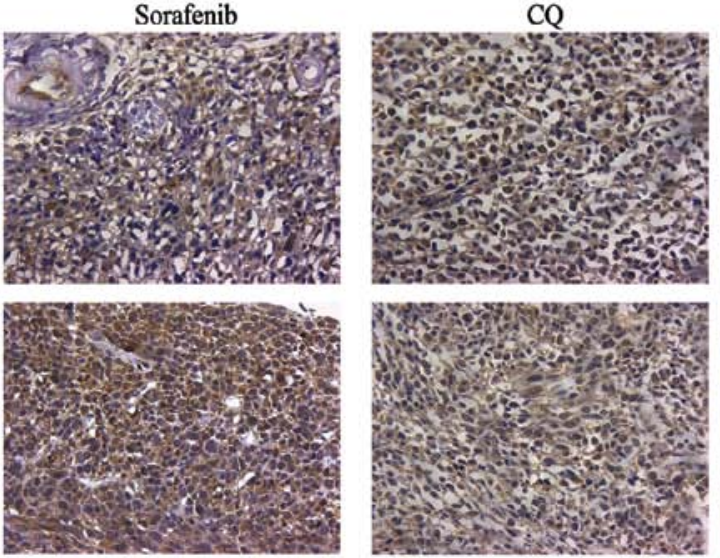

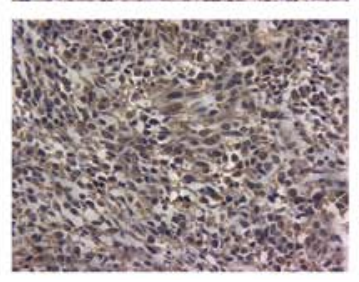

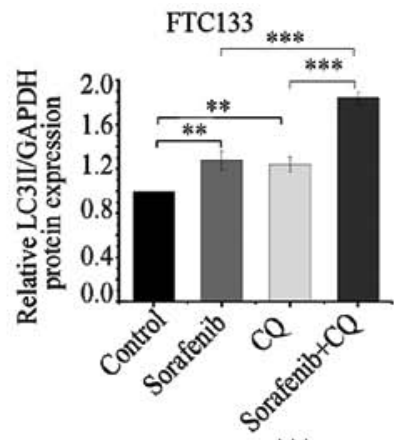

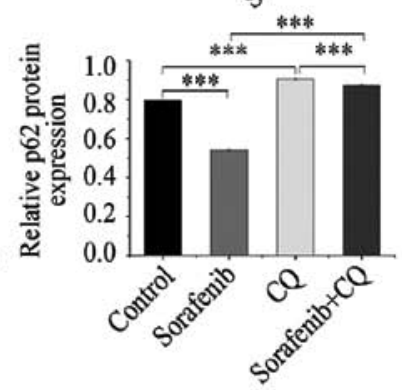

$\mathbf{F}$

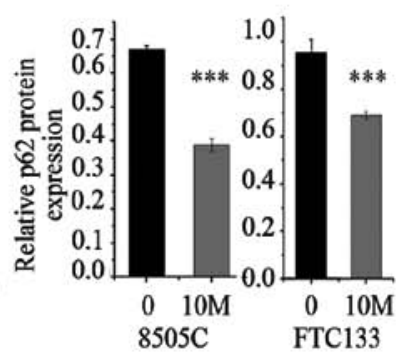

Figure 2. Sorafenib induces autophagy and apoptosis, and combined CQ and sorafenib treatment increases autophagy and apoptosis levels. (A) Tumors were lysed and western blot analyses of protein expression were performed. (B and C) Relative expression levels of LC3II/GAPDH and p62/GAPDH were analyzed by ImageJ densitometric analysis. Each band was measured three times. (D) Western blot analysis of 8505C and FTC133 cells treated with increasing concentrations of sorafenib. (E and F) Relative levels of LC3II/GAPDH and p62/GAPDH were analyzed by ImageJ densitometric analysis. Each band was measured three times. (G) Representative images from tumor sections stained for cleaved-caspase-3 (magnification, $\mathrm{x} 200) .{ }^{* *} \mathrm{P}<0.01$ and ${ }^{* * * *} \mathrm{P}<0.001$.

Sorafenib induces autophagy and apoptosis, and combined treatment with $C Q$ increases autophagy and apoptosis. $8505 \mathrm{C}$ and FTC133 xenograft mice were sacrificed and the tumor tissues were isolated for further studies after 21 days of treatment. The proteins LC3 and p62 were used as autophagy markers for monitoring autophagic flux and were examined by western blotting of lysed tumor tissues. The expression level of LC3II/GAPDH increased significantly in the sorafenib treatment group compared with the control group, while the expression level of p62 was reduced in the sorafenib treatment group compared with the control group (Fig. 2A-C). To confirm the effect of sorafenib on autophagy in thyroid cancer, we treated $8505 \mathrm{C}$ and FTC133 cell lines with various concentrations of sorafenib in vitro for $24 \mathrm{~h}$. With 
A
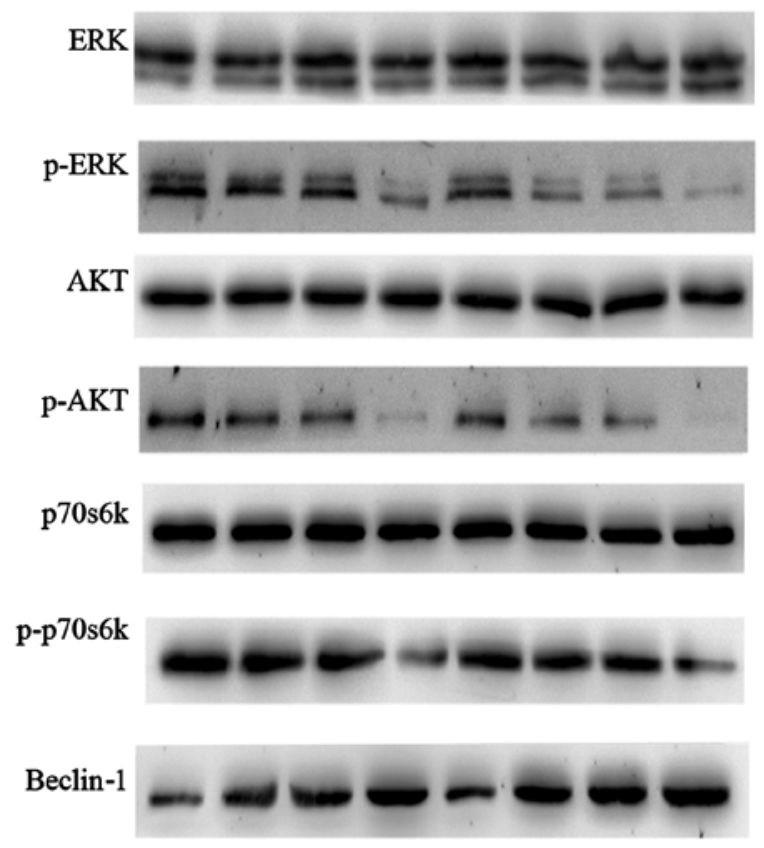

GAPDH

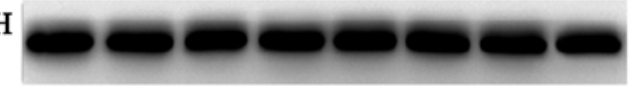

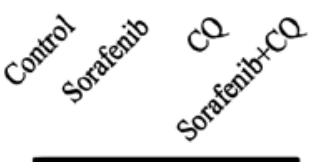

$8505 \mathrm{C}$

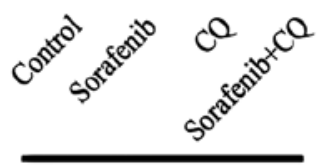

FTC133

\section{B}
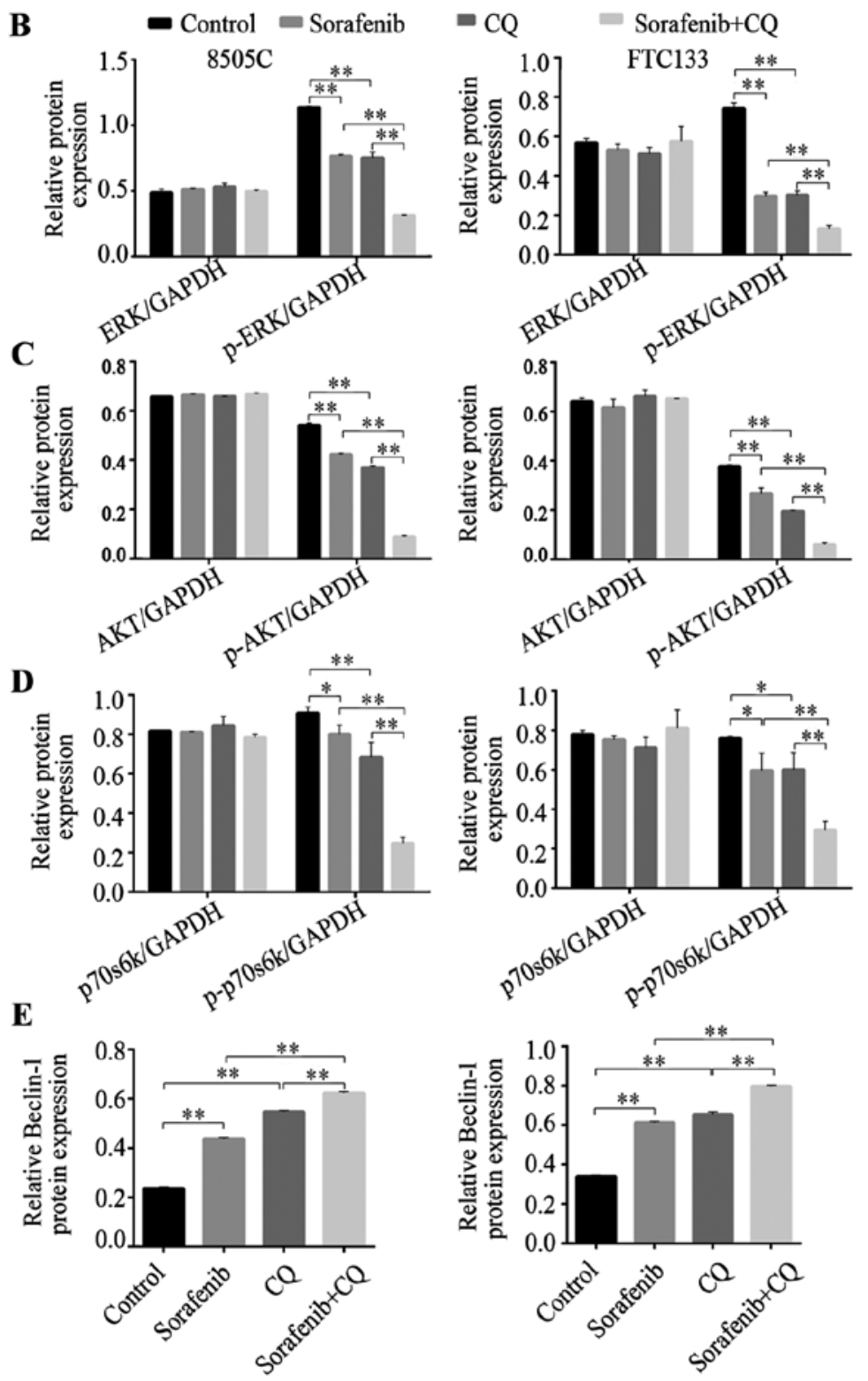

Figure 3. Sorafenib concurrently inhibits the activities of the MAPK and AKT/mTOR pathways in thyroid cancer. (A) Tumors were lysed and western blot analyses of protein expression was performed. (B-E) Relative levels of ERK/GAPDH, p-ERK/ERK, AKT/GAPDH, p-AKT/AKT, p70s6k/GAPDH, p-p70s6k/p70s6k, and Beclin-1/GAPDH were analyzed by ImageJ densitometric analysis. Each band was measured three times. ${ }^{*}<0.05$ and ${ }^{* *} \mathrm{P}<0.01$.

an increase in sorafenib concentration, LC3II/GAPDH levels increased significantly compared with the control. When treated with $10 \mu \mathrm{M}$ sorafenib, p62 protein levels were obviously reduced compared with the control (Fig. 2D-F). These results indicated that sorafenib increased autophagic flux in $8505 \mathrm{C}$ and FTC133 xenograft mouse tumors and in vitro experiments. In CQ-treated tumor tissues, the expression level of LC3II/GAPDH increased significantly compared with the control. However, the expression level of p62 in the CQ treatment group increased significantly compared with the control group, which appeared to be contrary to the sorafenib group (Fig. 2A-C). These results indicated that autophagic flux was inhibited by $\mathrm{CQ}$.

Caspase-3 is activated in apoptotic cells by both extrinsic and intrinsic pathways $(21,22)$. We used caspase- 3 activation to assess apoptosis since caspase- 3 is an early marker for apoptosis (23). To assess the effect of sorafenib and CQ on tumor apoptosis, we used IHC analysis of cleaved caspase-3 in different treatment groups of mice. As expected, in the sorafenib- and CQ-treated groups, the level of cleaved caspase-3 increased compared with the control, suggesting CQ or sorafenib induced apoptosis in 8505C and FTC133 xenograft mouse tumors. In the $\mathrm{CQ}+$ sorafenib combined treatment group, a further increase of cleaved caspase- 3 was observed compared with CQ- or sorafenib-treated groups in both $8505 \mathrm{C}$ and FTC133 xenograft mice (Fig. 2G). Our results demonstrated an activation of caspase-3 in 8505C and FTC133 xenograft mouse tumors, suggesting that caspase-mediated apoptosis occurs following sorafenib and CQ treatment.

Sorafenib concurrently inhibits activity of the MAPK and AKT/mTOR pathways in thyroid cancer. Tumor tissues were used to analyze the target-suppressing effects of sorafenib. As shown in Fig. 3A-D, compared with the control groups, the 
A

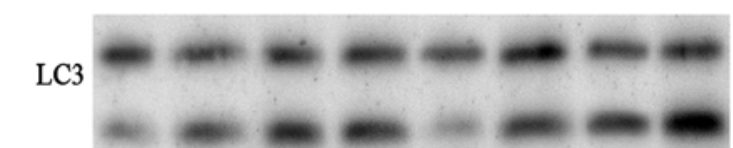

p62

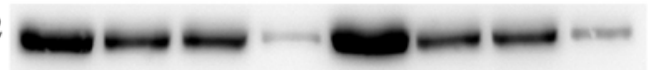

GAPDH

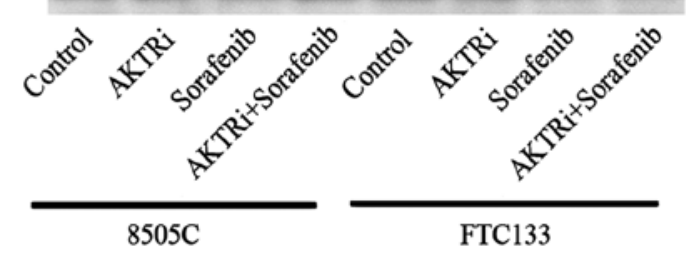

D

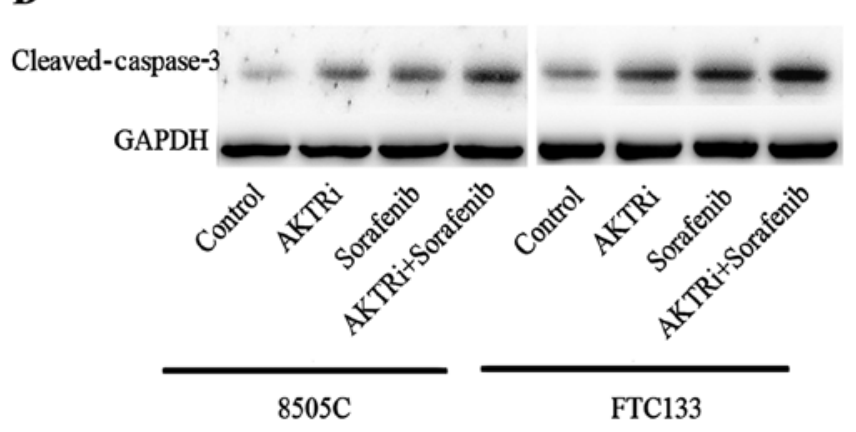

B
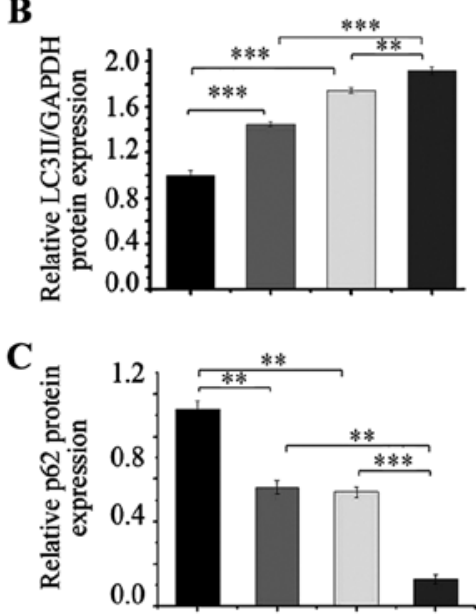

$\mathbf{E}$

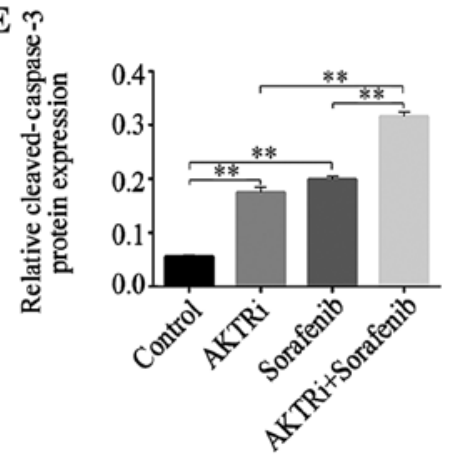

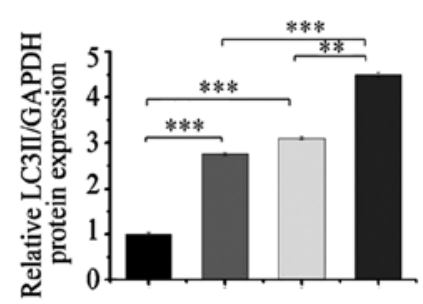
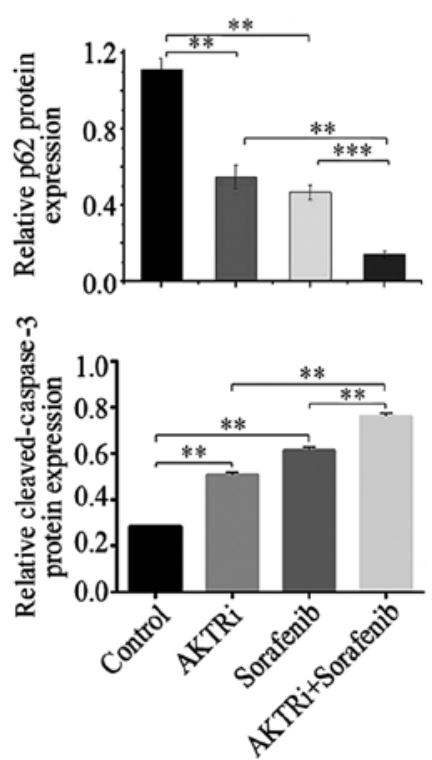

Figure 4. Inhibiting the AKT/mTOR pathway activates autophagy and apoptosis, and enhances sorafenib-induced autophagy and apoptosis. 8505C and FTC133 cells were treated with AKTRi mimic, sorafenib, or AKTRi mimic + sorafenib for 24 h. (A) Western blot analysis was performed to detect the levels of autophagy-associated proteins. (B and C) Relative levels of LC3II/GAPDH and p62/GAPDH were analyzed by ImageJ densitometric analysis. Each band was measured three times. (D) Western blot analysis was performed to detect the expression of cleaved-caspase-3 protein. (E) Relative quantity of cleavedcaspase-3/GAPDH was analyzed by ImageJ densitometric analysis. Each band was measured three times. ${ }^{* *} \mathrm{P}<0.01$ and ${ }^{* * * *} \mathrm{P}<0.001$.

expression of the ERK protein was not significantly different in the sorafenib treatment groups of $8505 \mathrm{C}$ and FTC133 xenograft mice. However, phosphorylation levels of ERK were significantly inhibited in both $8505 \mathrm{C}$ and FTC133 xenograft mice that were treated with sorafenib. Furthermore, we observed that the phosphorylation levels of AKT and p70s6k were significantly reduced in the sorafenib treatment groups compared with control groups. The results indicated that CQ exerted similar effects to sorafenib. The phosphorylation levels of ERK, AKT and p70s6k were significantly reduced after CQ treatment. Furthermore, in 8505C and FTC133 xenograft mice treated with combined CQ + sorafenib, the dephosphorylation effect was enhanced compared with that achieved by either drug alone (Fig. 3A-D).

Beclin-1 is a key autophagy-related gene that takes part in the initial process of autophagy (24). Beclin-1 protein expression was increased in xenograft mice treated with CQ or sorafenib, while combined CQ + sorafenib treatment increased the expression of Beclin-1 protein more than CQ or sorafenib treatment alone (Fig. 3A and E).

Inhibition of the AKT/mTOR pathway activates autophagy and apoptosis, and enhances the effect of sorafenib on autophagy and apoptosis induction. The MAPK pathway is a well-known target of sorafenib. However, the AKT/mTOR pathway was inhibited by sorafenib in the present study. The
AKT/mTOR pathway is a major pathway that regulates the process of autophagy $(25,26)$. Next, we demonstrated the role of the AKT/mTOR pathway in sorafenib-treated thyroid cell lines. To construct an AKT gene-silencing cell model, we transfected siRNAs into $8505 \mathrm{C}$ and FTC133 cell lines. The silencing effect was examined by western blotting. The protein levels of p-AKT were reduced significantly in AKT siRNAtransfected cell line models (27). The LC3 and p62 proteins were examined again for the evaluation of autophagy levels. Following silencing of AKT by siRNA, LC3II increased and p62 decreased compared with the control group (Fig. 4A-C). AKT silencing in combination with sorafenib treatment significantly enhanced LC3II expression levels and reduced p62 compared with AKT silencing or sorafenib treatment alone (Fig. 4A-C). BEZ-235, a dual, ATP-competitive PI3K and mTOR inhibitor, was selected to demonstrate the role of the AKT/mTOR pathway in sorafenib-induced autophagy. In a previous study, $0.8 \mu \mathrm{M}$ BEZ-235 inhibited AKT/mTOR pathway activity and inhibited $8505 \mathrm{C}$ and FTC133 cell line proliferation (27). Thus, 8505C and FTC133 cell lines were treated with $0.8 \mu \mathrm{M}$ BEZ-235 for $24 \mathrm{~h}$, and western blotting was used to evaluate the expression levels of LC3II and p62. LC3II expression increased and p62 expression decreased compared with the control group in both $8505 \mathrm{C}$ and FTC133 cell lines (data not shown). Combined treatment with sorafenib and BEZ-235 significantly enhanced LC3II expression and 
A

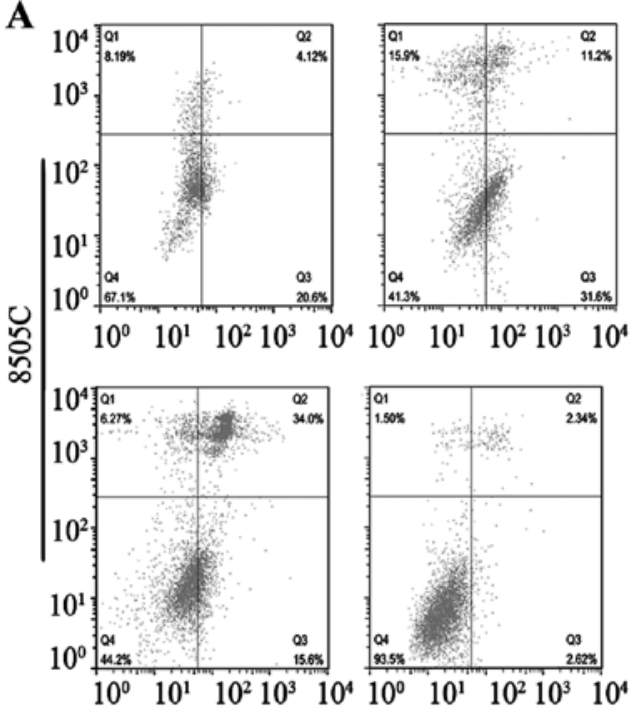

C

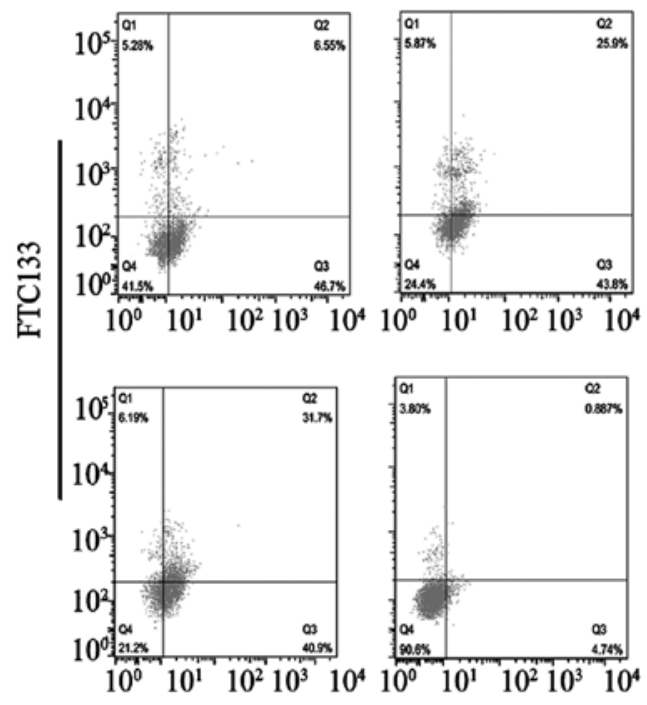

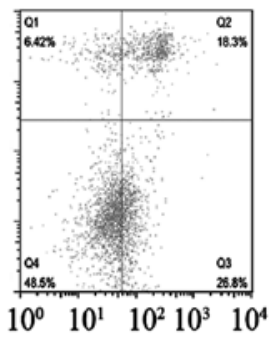
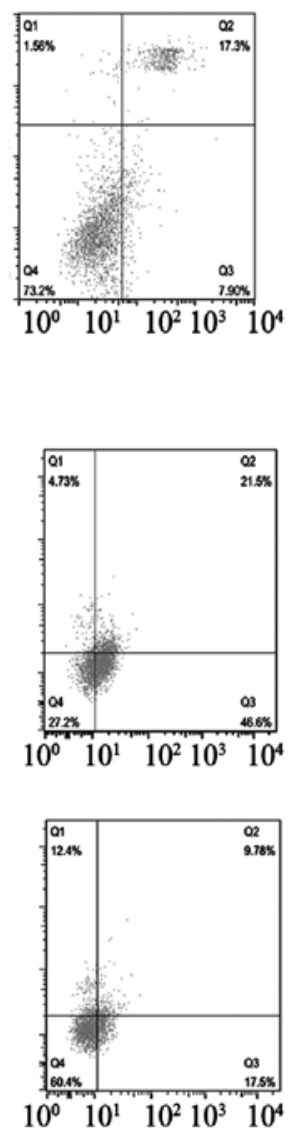

B

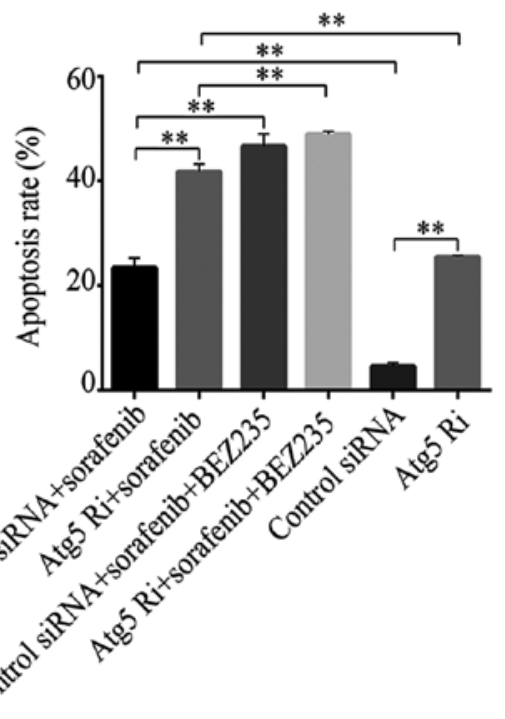

D

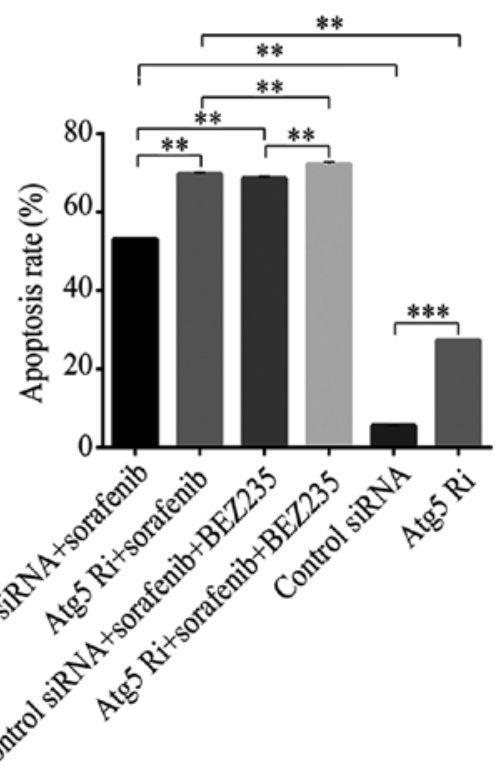

Figure 5. Inhibition of autophagy enhances sorafenib-induced apoptosis in thyroid cancer cell lines. 8505C and FTC133 cell lines were incubated with ATG5Ri mimic, sorafenib $(10 \mu \mathrm{M})$, BEZ-235 $(0.8 \mu \mathrm{M})$ or combined sorafenib + BEZ-235. (A and C) The cells were analyzed by flow cytometry to detect apoptosis. (B and D) The rates of apoptosis were determined. Data represent three independent experiments. ${ }^{* *} \mathrm{P}<0.01$ and ${ }^{* * *} \mathrm{P}<0.001$.

reduced p62 expression (data not shown). The AKT/mTOR pathway negatively regulates autophagy and apoptosis (17). In the present study, 8505C and FTC133 cell lines treated with AKT siRNA exhibited increased cleaved capase-3 expression, and combined treatment of AKT siRNA with sorafenib enhanced cleaved capase-3 expression compared with either treatment alone (Fig. 4D and E).

Effect of autophagy on sorafenib treated thyroid cancer cell lines. In our results, sorafenib treatment or AKT/mTOR pathway suppression induced autophagy in thyroid cancer cell lines. As autophagy plays a dual role in cancer treatment, we examined the role of autophagy in sorafenib treatment of thyroid cancer $(11,12)$. ATG5 is a key gene in the process of autophagy that can be silenced to demonstrate the role of autophagy in different treatments (6). Three different siRNAs (ATG5Ri) were transfected into 8505C and FTC133 cell lines, and RT-qPCR was used to evaluate their silencing effect. ATG5 siRNA2 appeared to have strong silencing effects and was selected for use in further experiments (data not shown).

Treatment with sorafenib or ATG5Ri increased the apoptosis of 8505C and FTC133 cell lines compared with the control group (apoptosis rate, 4.9 and 5.6\%); the apoptosis rates after sorafenib or ATG5Ri treatment were 24.7 and $25.2 \%$, respectively, in $8505 \mathrm{C}$ cells (Fig. 5A and B), and 53.2 and $27.3 \%$, respectively, in FTC133 cells (Fig. 5C and D). Combined treatment with sorafenib and ATG5Ri increased apoptosis rates more than either treatment alone in both cell lines $(42.8 \%$ in $8505 \mathrm{C}$ cells; $69.7 \%$ in FTC133 cells; Fig. 5). Silencing of ATG5 significantly increased the apoptosis rate after combined treatment of sorafenib and BEZ235 in FTC133 cells (72.6 vs. 68.1\%; Fig. 5). Silencing of ATG5 increased the anti-proliferative effect of sorafenib and combined treatment of sorafenib and BEZ-235 in both $8505 \mathrm{C}$ and FTC133 cell lines (Fig. 6). 
A

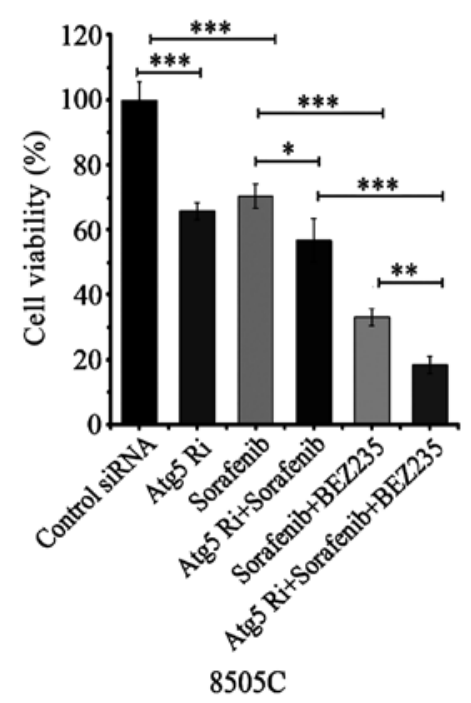

B

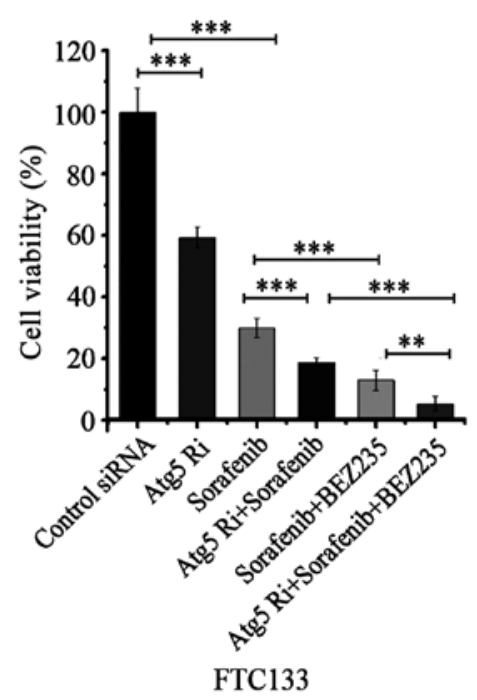

Figure 6. Silencing of ATG5 increases the anti-proliferative effect of sorafenib in thyroid cancer cell lines. 8505C and FTC133 cell lines were incubated with ATG5Ri mimic, sorafenib $(10 \mu \mathrm{M})$, BEZ-235 $(0.8 \mu \mathrm{M})$ or combined treatments for $48 \mathrm{~h}$. Cell viability was calculated using a CCK-8 kit. ${ }^{*} \mathrm{P}<0.05,{ }^{* *} \mathrm{P}<0.01$ and ${ }^{* * *} \mathrm{P}<0.001$.

\section{Discussion}

Resistance to ${ }^{131}$ iodine therapy is an important factor leading to poor prognosis for DTC patients $(2,3)$. Several targeted kinase inhibitors have been used in clinical trials and administered to patients who have advanced/progressive ${ }^{131}$ iodine-refractory DTC disease (28). Sorafenib targets the RAF serine/threonine kinases, and also potently inhibits tyrosine kinase receptors, such as vascular endothelial growth factor receptor and platelet-derived growth factor receptor- $\beta$, which promote angiogenesis $(7,8)$. It has been reported that sorafenib has therapeutic effects in several types of cancer, such as HCC, acute myeloid leukemia, advanced renal cell carcinoma or prostate cancer (29-32). Sorafenib is the first tyrosine kinase inhibitor (TKI) approved for advanced/progressive ${ }^{131}$ iodinerefractory DTC patients in China. After sorafenib treatment, the majority of advanced/progressive ${ }^{131}$ iodine-refractory DTC patients exhibit a favorable prognosis. However, most patients acquire resistance to sorafenib after 1 or 2 years of administration, and the mechanism of this acquired resistance remains unclear (10). In the treatment of various cancers, including liver, kidney and prostate cancer, sorafenib can achieve a few months of progression-free survival, but drug resistance frequently emerges (31-33). Mechanisms of sorafenib resistance may be related to EGFR activation, downstream signaling of EGFR (particularly Ras/Raf/MEK/ERK), the PI3K/AKT pathway, autophagy or epithelial-mesenchymal transition, as reviewed by Beardsley et al (32), and Chen et al (33).

The process of autophagy could be activated during cancer treatment by radiation (34), chemotherapy (35) or TKI drugs $(12,15)$. The role of autophagy in cancer treatment is complex since it is a 'double-edged sword'; autophagy may promote or inhibit cell death $(12,36,37)$. The varying roles of autophagy in cancer treatment primarily depend on different contexts and treatments $(13,37,38)$. In different HCC cell lines, the role of autophagy induced by sorafenib was reversed, as detailed by Prieto-Dominguez et al (13). It has been reported that inhibition of AKT reverses acquired resistance to sorafenib by switching protective autophagy to autophagic cell death (17).

It has been demonstrated that sorafenib triggers autophagy in different cancer contexts $(12,15)$. In the present study, sorafenib induced autophagy in thyroid cancer cell lines and mice xenograft models. Inhibition of autophagy by either a pharmacological inhibitor (CQ) or siRNA knockdown of essential autophagy genes increased the effect of sorafenib treatment. CQ has been safely used for decades in patients for malaria prophylaxis and for the treatment of rheumatoid arthritis (39). The anticancer role of CQ has primarily been attributed to its autophagy regulation role. Autophagy is an evolutionarily conserved cellular degradation process that engulfs cytoplasmic materials in double-membrane structures (autophagosomes), which are subsequently transported to lysosomes for degradation and recycling $(11,40)$. CQ is an autophagy inhibitor that prevents autophagosomes from fusing with lysosomes, thereby disrupting the process of autophagy (20). The primary role of CQ in cancer treatment is to enhance the efficacy of a drug or other treatment measure (41). However, CQ does not markedly alter anticancer efficiency in certain types of cancer; it may depend on the tumor type and context (42). To the best of our knowledge, no previous studies have demonstrated the role of CQ in sorafenib treatment of thyroid cancer. In the present study, thyroid cancer xenograft mice were administered CQ. The results indicated that the processes of autophagy were inhibited by $\mathrm{CQ}$, and that the anticancer efficiency of sorafenib was significantly increased. Recent studies have indicated that the synergistic effects of CQ in cancer treatment are independent of autophagy in several types of cancer (43-45). The mechanism of the synergistic effects of CQ is consistent with lysosomal cell death (43), vessel normalization (44) and other mechanisms (45). In order to confirm the role of autophagy in sorafenib treatment of thyroid cancer, further regulatory pathways and autophagy genes were evaluated. 
Activation of the PI3K/AKT signaling pathway mediates acquired resistance to sorafenib in HCC (46). Sorafenib has been reported to inhibit both the MAPK and AKT/mTOR pathways in lymphoma xenografts (47). Similarly, in the present study, sorafenib inhibited both the MAPK and AKT/mTOR pathways. mTOR is a negative regulator of autophagy and promotes the proliferation of cancer cells (18). In the present study, the AKT/mTOR pathway was selected to demonstrate the roles of autophagy in thyroid cancer treatment by sorafenib. We silenced the AKT/mTOR pathway by siRNA technology or with an inhibitor (BEZ-235 is a dual inhibitor of PI3K and AKT). Subsequent results indicated that the downstream gene activity of the AKT/mTOR pathway was suppressed, cell proliferation was significantly reduced, and apoptosis and autophagy were enhanced. These results indicated that sorafenib induced autophagy, at least in part due to inhibition of the AKT/mTOR pathway.

Beclin-1 is a key gene participating in the initial process of autophagy (48). The expression of Beclin-1 was promoted after sorafenib treatment in the present study. This finding indicates that a complex mechanism participates in sorafenibinduced autophagy. However, due to the dual role of autophagy in cancer treatment, it is not clear whether sorafenib-induced autophagy or AKT/mTOR pathway inhibition promote or inhibit cell death (37).

ATG5 is an E3 ubiquitin ligase that is critical for autophagy due to its role in autophagosome elongation (6). Silencing of ATG5 demonstrates the role of autophagy in a cancer treatment process (6). Treatment with ATG5 siRNA in the present study induced apoptosis in $8505 \mathrm{C}$ and FTC133 cell lines. Combined treatment with an ATG5 siRNA increased the tumor-suppressive effect of sorafenib in $8505 \mathrm{C}$ and FTC133 cell lines. The apoptosis rate of the ATG5 silencing + sorafenib + BEZ235 treatment group increased more than that of the sorafenib + BEZ235 treatment group in FTC133 cells. We did not observe a significantly different apoptosis rate between the ATG5 silencing + sorafenib + BEZ-235 treatment group and the sorafenib + BEZ-235 treatment group in $8505 \mathrm{C}$ cells. In our data, silenced ATG5 suppressed the proliferation of 8505C and FTC133 cells, and ATG5 silencing combined with sorafenib treatment significantly suppressed cell proliferation in $8505 \mathrm{C}$ and FTC133 cells. Our previous study indicated that silencing the AKT/mTOR pathway induced apoptosis, suppressed proliferation and induced autophagy in both 8505C and FTC133 cells (27). In the present study, sorafenib inhibited both the MAPK and AKT/mTOR pathways in $8505 \mathrm{C}$ and FTC133 cells. Thus, AKT/mTOR inhibition by sorafenib represents a positive effect of sorafenib treatment in thyroid cancer. However, autophagy induced by sorafenib involves a complex mechanism and can only be partially attributed to AKT/mTOR suppression. Comprehensive analysis of our results revealed that autophagy is a protective mechanism in sorafenib treatment of thyroid cancer. Our study had limitations. The additive or synergistic effect of sorafenib and CQ in mouse xenograft models can not be easily established and can be examined by future studies.

In summary, the present study demonstrated that sorafenib induces autophagy in thyroid cancer, and that this effect is, at least in part, due to AKT/mTOR pathway suppression. We demonstrated that CQ increased the efficacy of sorafenib in treating thyroid cancer in xenograft mice, resulting in decreased tumor growth. Treatment with CQ or ATG5 silencers blocked the processes of autophagy and increased apoptosis in sorafenib-treated tumors. Autophagy has a cytoprotective role in sorafenib treatment of thyroid cancer. Combined treatment of sorafenib with CQ or other autophagy inhibitors is a novel and potentially useful clinical strategy to improve the efficacy of sorafenib-targeted thyroid cancer therapies. Our findings provide a basis for investigating the use of sorafenib with autophagy inhibitors to treat ${ }^{131}$ iodine-resistant, advanced DTC.

\section{Acknowledgements}

The present study was supported by the Zhejiang Provincial Natural Science Foundation of China (grant no.LY15H180002), and the Medical and Health Research Program of Zhejiang Province (grant no. 2015DTA003).

\section{References}

1. Chen W, Zheng R, Baade PD, Zhang S, Zeng H, Bray F, Jemal A, Yu XQ and He J: Cancer statistics in China, 2015. CA Cancer J Clin 66: 115-132, 2016.

2. Song HJ, Qiu ZL, Shen CT, Wei WJ and Luo QY: Pulmonary metastases in differentiated thyroid cancer: Efficacy of radioiodine therapy and prognostic factors. Eur J Endocrinol 173: 399-408, 2015.

3. Durante C, Haddy N, Baudin E, Leboulleux S, Hartl D, Travagli JP, Caillou B, Ricard M, Lumbroso JD, De Vathaire F, et al: Long-term outcome of 444 patients with distant metastases from papillary and follicular thyroid carcinoma: Benefits and limits of radioiodine therapy. J Clin Endocrinol Metab 91: 2892-2899, 2006.

4. Vogelstein B, Papadopoulos N, Velculescu VE, Zhou S, Diaz LA Jr and Kinzler KW: Cancer genome landscapes. Science 339: 1546-1558, 2013.

5. Xing M, Haugen BR and Schlumberger M: Progress in molecularbased management of differentiated thyroid cancer. Lancet 381: 1058-1069, 2013.

6. Wang W, Kang H, Zhao Y, Min I, Wyrwas B, Moore M, Teng L, Zarnegar R, Jiang $X$ and Fahey TJ III: Targeting autophagy sensitizes BRAF-mutant thyroid cancer to vemurafenib. J Clin Endocrinol Metab 102: 634-643, 2017.

7. Wilhelm SM, Adnane L, Newell P, Villanueva A, Llovet JM and Lynch M: Preclinical overview of sorafenib, a multikinase inhibitor that targets both Raf and VEGF and PDGF receptor tyrosine kinase signaling. Mol Cancer Ther 7: 3129-3140, 2008.

8. Adnane L, Trail PA, Taylor I and Wilhelm SM: Sorafenib (BAY 43-9006, Nexavar), a dual-action inhibitor that targets RAF/MEK/ERK pathway in tumor cells and tyrosine kinases VEGFR/PDGFR in tumor vasculature. Methods Enzymol 407: 597-612, 2006.

9. Brose MS, Nutting CM, Jarzab B, Elisei R, Siena S, Bastholt L, de la Fouchardiere C, Pacini F, Paschke R, Shong YK, et al; DECISION investigators: Sorafenib in radioactive iodinerefractory, locally advanced or metastatic differentiated thyroid cancer: A randomised, double-blind, phase 3 trial. Lancet 384: 319-328, 2014.

10. Pitoia F and Jerkovich F: Selective use of sorafenib in the treatment of thyroid cancer. Drug Des Devel Ther 10: 1119-1131, 2016.

11. Yi H, Long B, Ye X, Zhang L, Liu X and Zhang C: Autophagy: A potential target for thyroid cancer therapy (Review). Mol Clin Oncol 2: 661-665, 2014.

12. Heqing Y, Bin L, Xuemei Y and Linfa L: The role and mechanism of autophagy in sorafenib targeted cancer therapy. Crit Rev Oncol Hematol 100: 137-140, 2016.

13. Prieto-Domínguez N, Ordóñez R, Fernández A, GarcíaPalomo A, Muntané J, González-Gallego J and Mauriz JL: Modulation of autophagy by sorafenib: Effects on treatment response. Front Pharmacol 7: 151, 2016. 
14. Shi YH, Ding ZB, Zhou J, Hui B, Shi GM, Ke AW, Wang XY, Dai Z, Peng YF, Gu CY, et al: Targeting autophagy enhances sorafenib lethality for hepatocellular carcinoma via ER stressrelated apoptosis. Autophagy 7: 1159-1172, 2011.

15. Shimizu S, Takehara T, Hikita H, Kodama T, Tsunematsu H, Miyagi T, Hosui A, Ishida H, Tatsumi T, Kanto T, et al: Inhibition of autophagy potentiates the antitumor effect of the multikinase inhibitor sorafenib in hepatocellular carcinoma. Int J Cancer 131: 548-557, 2012

16. Yuan H, Li AJ, Ma SL, Cui LJ, Wu B, Yin L and Wu MC: Inhibition of autophagy significantly enhances combination therapy with sorafenib and HDAC inhibitors for human hepatoma cells. World J Gastroenterol 20: 4953-4962, 2014.

17. Zhai B, Hu F, Jiang X, Xu J, Zhao D, Liu B, Pan S, Dong X, Tan $\mathrm{G}$, Wei $\mathrm{Z}$, et al: Inhibition of Akt reverses the acquired resistance to sorafenib by switching protective autophagy to autophagic cell death in hepatocellular carcinoma. Mol Cancer Ther 13: 1589-1598, 2014.

18. Yu L, McPhee CK, Zheng L, Mardones GA, Rong Y, Peng J, Mi N, Zhao Y, Liu Z, Wan F, et al: Termination of autophagy and reformation of lysosomes regulated by mTOR. Nature 465: 942-946, 2010.

19. Zou Y, Ling YH, Sironi J, Schwartz EL, Perez-Soler R and Piperdi B: The autophagy inhibitor chloroquine overcomes the innate resistance of wild-type EGFR non-small-cell lung cancer cells to erlotinib. J Thorac Oncol 8: 693-702, 2013.

20. Wang C, Hu Q and Shen H-M: Pharmacological inhibitors of autophagy as novel cancer therapeutic agents. Pharmacol Res 105: 164-175, 2016.

21. Salvesen GS: Caspases: Opening the boxes and interpreting the arrows. Cell Death Differ 9: 3-5, 2002.

22. Ghavami S, Hashemi M, Ande SR, Yeganeh B, Xiao W, Eshraghi M, Bus CJ, Kadkhoda K, Wiechec E, Halayko AJ, et al: Apoptosis and cancer: Mutations within caspase genes. J Med Genet 46: 497-510, 2009.

23. Günther A, Luczak V, Abel T and Baumann A: Caspase-3 and GFAP as early markers for apoptosis and astrogliosis in shRNAinduced hippocampal cytotoxicity. J Exp Biol 220: 1400-1404, 2017.

24. Zhong Y, Wang QJ, Li X, Yan Y, Backer JM, Chait BT, Heintz N and Yue Z: Distinct regulation of autophagic activity by Atg14L and Rubicon associated with Beclin 1-phosphatidylinositol3-kinase complex. Nat Cell Biol 11: 468-476, 2009.

25. Vlahakis A and Powers T: A role for TOR complex 2 signaling in promoting autophagy. Autophagy 10: 2085-2086, 2014.

26. Díaz-Troya S, Pérez-Pérez ME, Florencio FJ and Crespo JL: The role of TOR in autophagy regulation from yeast to plants and mammals. Autophagy 4: 851-865, 2008.

27. Yi H, Ye X, Long B, Ye T, Zhang L, Yan F, Yang Y and Li L: Inhibition of the AKT/mTOR pathway augments the anticancer effects of sorafenib in thyroid cancer. Cancer Biother Radiopharm 32: 176-183, 2017.

28. Valerio L, Pieruzzi L, Giani C, Agate L, Bottici V, Lorusso L, Cappagli V, Puleo L, Matrone A, Viola D, et al: Targeted therapy in thyroid cancer: State of the art. Clin Oncol (R Coll Radiol) 29: 316-324, 2017

29. Bruix J, Takayama T, Mazzaferro V, Chau GY, Yang J, Kudo M, Cai J, Poon RT, Han KH, Tak WY, et al; STORM investigators: Adjuvant sorafenib for hepatocellular carcinoma after resection or ablation (STORM): A phase 3, randomised, double-blind, placebo-controlled trial. Lancet Oncol 16: 1344-1354, 2015.

30. Tarlock K, Chang B, Cooper T, Gross T, Gupta S, Neudorf S, Adlard K, Ho PA, McGoldrick S, Watt T, et al: Sorafenib treatment following hematopoietic stem cell transplant in pediatric FLT3/ITD acute myeloid leukemia. Pediatr Blood Cancer 62: 1048-1054, 2015.

31. Hutson TE, Escudier B, Esteban E, Bjarnason GA, Lim HY, Pittman KB, Senico P, Niethammer A, Lu DR, Hariharan S, et al: Randomized phase III trial of temsirolimus versus sorafenib as second-line therapy after sunitinib in patients with metastatic renal cell carcinoma. J Clin Oncol 32: 760-767, 2014.
32. Beardsley EK, Hotte SJ, North S, Ellard SL, Winquist E, Kollmannsberger C, Mukherjee SD and Chi KN: A phase II study of sorafenib in combination with bicalutamide in patients with chemotherapy-naive castration resistant prostate cancer. Invest New Drugs 30: 1652-1659, 2012.

33. Cheng AL, Kang YK, He AR, Lim HY, Ryoo BY, Hung CH, Sheen IS, Izumi N, Austin T, Wang Q, et al; Investigators' Study Group: Safety and efficacy of tigatuzumab plus sorafenib as firstline therapy in subjects with advanced hepatocellular carcinoma: A phase 2 randomized study. J Hepatol 63: 896-904, 2015.

34. Hu L, Wang H, Huang L, Zhao Y and Wang J: Crosstalk between autophagy and intracellular radiation response (Review). Int J Oncol 49: 2217-2226, 2016.

35. Liang B, Liu X, Liu Y, Kong D, Liu X, Zhong R and Ma S: Inhibition of autophagy sensitizes MDR-phenotype ovarian cancer SKVCR cells to chemotherapy. Biomed Pharmacother 82: 98-105, 2016.

36. Kondo Y, Kanzawa T, Sawaya R and Kondo S: The role of autophagy in cancer development and response to therapy. Nat Rev Cancer 5: 726-734, 2005.

37. Thorburn A, Thamm DH and Gustafson DL: Autophagy and cancer therapy. Mol Pharmacol 85: 830-838, 2014.

38. White E: The role for autophagy in cancer. J Clin Invest 125 : 42-46, 2015.

39. O'Neill PM, Bray PG, Hawley SR, Ward SA and Park BK: 4-Aminoquinolines--past, present, and future: A chemical perspective. Pharmacol Ther 77: 29-58, 1998.

40. Yin Z, Pascual C and Klionsky DJ: Autophagy: Machinery and regulation. Microb Cell 3: 588-596, 2016.

41. Grimaldi A, Santini D, Zappavigna S, Lombardi A, Misso G, Boccellino M, Desiderio V, Vitiello PP, Di Lorenzo G, Zoccoli A, et al: Antagonistic effects of chloroquine on autophagy occurrence potentiate the anticancer effects of everolimus on renal cancer cells. Cancer Biol Ther 16: 567-579, 2015

42. Zinn RL, Gardner EE, Dobromilskaya I, Murphy S, Marchionni L Hann CL and Rudin CM: Combination treatment with ABT-737 and chloroquine in preclinical models of small cell lung cancer. Mol Cancer 12: 16, 2013.

43. King MA, Ganley IG and Flemington V: Inhibition of cholesterol metabolism underlies synergy between mTOR pathway inhibition and chloroquine in bladder cancer cells. Oncogene 35: 4518-4528, 2016.

44. Maes H, Kuchnio A, Peric A, Moens S, Nys K, De Bock K, Quaegebeur A, Schoors S, Georgiadou M, Wouters J, et al: Tumor vessel normalization by chloroquine independent of autophagy. Cancer Cell 26: 190-206, 2014.

45. Chen X, Clark J, Wunderlich M, Fan C, Davis A, Chen S, Guan JL, Mulloy JC, Kumar A and Zheng Y: Autophagy is dispensable for Kmt2a/Mll-Mllt3/Af9 AML maintenance and anti-leukemic effect of chloroquine. Autophagy 13: 955-966, 2017.

46. Chen KF, Chen HL, Tai WT, Feng WC, Hsu CH, Chen PJ and Cheng AL: Activation of phosphatidylinositol 3-kinase/Akt signaling pathway mediates acquired resistance to sorafenib in hepatocellular carcinoma cells. J Pharmacol Exp Ther 337: 155-161, 2011.

47. Carlo-Stella C, Locatelli SL, Giacomini A, Cleris L, Saba E, Righi M, Guidetti A and Gianni AM: Sorafenib inhibits lymphoma xenografts by targeting MAPK/ERK and AKT pathways in tumor and vascular cells. PLoS One 8: e61603, 2013.

48. Liu G, Yuan Y, Long M, Luo T, Bian J, Liu X, Gu J, Zou H, Song R, Wang Y, et al: Beclin-1-mediated autophagy protects against cadmium-activated apoptosis via the Fas/FasL pathway in primary rat proximal tubular cell culture. Sci Rep 7: 977, 2017. 